\title{
Critical phenomenon in soft magnetic properties of giant magnetostrictive materials: Computer simulation
}

\author{
Hirotoshi Fukunaga a) and Takayuki Yamaguchi \\ Graduate School of Science and Technology, Nagasaki University, Nagasaki 852, Japan
}

(Presented on 2 November 2005; published online 27 April 2006)

\begin{abstract}
Dependence of magnetostrictive and magnetic properties on grain size was calculated numerically for an isotropic giant magnetostrictive material. The model magnet assumed is composed of 8000 cubic $\left(\mathrm{Tb}_{0.3} \mathrm{Dy}_{0.7}\right) \mathrm{Fe}_{2}$ grains. The direction of magnetization and the magnetostriction of each grain was determined for a given applied field under the consideration of the anisotropy, exchange, magnetoelastic, elastic, and Zeeman energies. Subsequently, the hysteresis loop and the magnetostriction versus applied field curve was obtained by varying the applied field. With decreasing the grain size $D$, the coercivity $H_{c}$ and $d \lambda / d H$, where $\lambda$ is the magnetostriction, decreased abruptly at $D=25 \mathrm{~nm}$. This improvement in soft magnetic properties exceeded the expected improvement from Herzer's calculation, in which $H_{c}$ was proportional to $D^{6}$. In order to study the significant improvement in detail, we calculated magnetic and magnetostrictive properties with varying magnetostriction and anisotropy constants. Resultantly, it was clarified that the critical phenomenon occurs when the total of elastic and magnetoelastic energies exceed the magnetic anisotropy energy in magnitude. (C) 2006 American Institute of Physics. [DOI: 10.1063/1.2176311]
\end{abstract}

Magnetostrictive materials require both giant saturation magnetostriction and soft magnetic properties. Although the cubic Laves phase $\mathrm{TbFe}_{2}$ has the largest magnetostriction at room temperature to date, ${ }^{1}$ it is magnetically hard because of its large crystalline magnetic anisotropy constant $K_{c 1}$ $=-6.3 \mathrm{MJ} / \mathrm{m}^{3}$. Clark et al. have succeeded in decreasing $K_{c 1}$ without a large reduction in saturation magnetostriction by the replacement of a part of Tb with Dy, and Terfenol-D $\left[\left(\mathrm{Tb}_{0.3} \mathrm{Dy}_{0.7}\right) \mathrm{Fe}_{2}\right]$ is widely known as one of the best magnetostrictive materials. ${ }^{1}$

Recently, it was reported that a refinement of the grain size improves soft magnetic properties of $\mathrm{Fe}-\mathrm{Si}-\mathrm{Nb}-\mathrm{Cu}-\mathrm{B}$ alloys significantly, ${ }^{2,3}$ and a similar effect has been confirmed experimentally for magnetostrictive alloys. ${ }^{4-7}$ However, the details of the relationship between grain size and soft magnetic properties are left unknown because of the difficulties in controlling the size and alignment of $\left(\mathrm{Tb}_{0.3} \mathrm{Dy}_{0.7}\right) \mathrm{Fe}_{2}$ grains.

In this contribution, we numerically studied the dependence of magnetostrictive and magnetic properties on grain size for isotropic polycrystalline $\left(\mathrm{Tb}_{0.3} \mathrm{Dy}_{0.7}\right) \mathrm{Fe}_{2}$ alloy by computer simulation, and found that soft magnetic properties are improved abruptly when the grain size is smaller than a critical value. This critical phenomenon was discussed in terms of energies stored in the material.

The model magnet assumed is composed of 8000 cubic grains in the single domain state as shown in Fig. 1. Each grain has the cubic magnetic anisotropy, and its crystal orientation was distributed randomly. Thus, the model is an isotropic polycrystalline magnet. The magnetization of a grain is coupled to those of six neighboring grains by the exchange interaction. The periodic boundary condition was used in the three directions.

\footnotetext{
${ }^{a}$ Electronic mail: fukunaga@net.nagasaki-u.ac.jp
}

The direction of magnetization and the magnetostriction of each grain as determined for a given applied field by minimizing the total energy stored in the model magnet. The total energy $W_{\text {total }}$ is given by the sum of the anisotropy, Zeeman, exchange, elastic, and magnetoelastic energies. The magnetostatic energy was neglected in this study, because its effect would be small compared to the other ones.

The anisotropy energy $W_{a}$ is written as

$$
W_{a}=V \sum_{i} K_{c 1}\left(\alpha_{1 i}^{\prime 2} \alpha_{2 i}^{\prime 2}+\alpha_{2 i}^{\prime 2} \alpha_{3 i}^{\prime 2}+\alpha_{3 i}^{\prime 2} \alpha_{1 i}^{\prime 2}\right),
$$

where $V$ and $K_{c 1}$ are the volume of the grain and the crystalline magnetic anisotropy constant, respectively. Furthermore, $\boldsymbol{\alpha}_{i}^{\prime}\left(\alpha_{1 i}^{\prime}, \alpha_{2 i}^{\prime}, \alpha_{3 i}^{\prime}\right)$ is the direction cosine vector of magnetization vector projected to the crystal axes of the $i$ th grain. The exchange energy $W_{\text {ex }}$ can be expressed by

$$
W_{\mathrm{ex}}=-V \sum_{i} \frac{A}{D^{2}}\left(\sum_{j}^{6} \boldsymbol{\alpha}_{i} \cdot \boldsymbol{\alpha}_{j}\right),
$$

where $A$ is the exchange stiffness constant, $D$ is the grain size, and $\boldsymbol{\alpha}_{i}\left(\alpha_{1 i}, \alpha_{2 i}, \alpha_{3 i}\right)$ is the direction cosine vector laying in the direction of the magnetization vector.

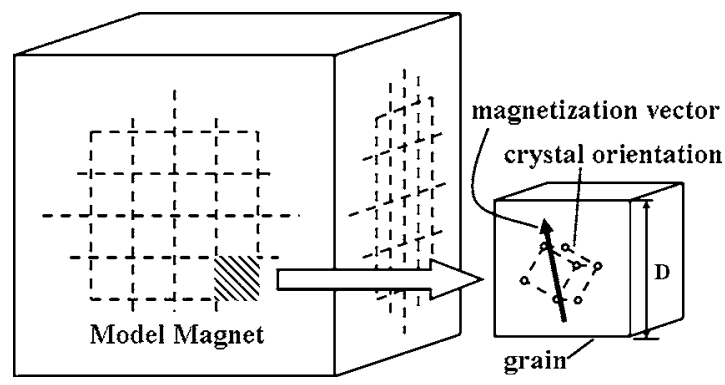

FIG. 1. Model magnet assumed. The magnet is composed of 8000 cubic $\left(\mathrm{Tb}_{0.3} \mathrm{Dy}_{0.7}\right) \mathrm{Fe}_{2}$ grains. 
TABLE I. Material parameters used in calculation.

\begin{tabular}{ccccc}
\hline \hline Material constants & $\left(\mathrm{Tb}_{0.3} \mathrm{Dy}_{0.7}\right) \mathrm{Fe}_{2}$ & Alloy 1 & Alloy 2-5 & Alloy 6 \\
\hline$K_{c 1}\left(\times 10^{5} \mathrm{~J} / \mathrm{m}^{3}\right)$ & 5.48 & 5.48 & 0.0 to 10.96 & 5.48 \\
$I_{s}(\mathrm{~T})$ & 1.0 & 1.0 & 1.0 & $\cdots$ \\
$\lambda_{100}, \lambda_{111}(\mathrm{ppm})$ & 90,1640 & 0,0 & variable & 1000,1000 \\
$c_{11}, c_{12}, c_{44}\left(\times 10^{10} \mathrm{~N} / \mathrm{m}\right)$ & $14.1,6.48,4.87$ & $\cdots$ & $14.1,6.48,4.87$ & $9.74,0.0,4.87$ \\
$A\left(\times 10^{-11} \mathrm{~J} / \mathrm{m}\right)$ & 2.5 & 2.5 & $\cdots$ & $\cdots$ \\
$D(\mathrm{~nm})$ & variable & variable & $\cdots$ & $\cdots$ \\
\hline \hline
\end{tabular}

In this study, all the grains were assumed to have the same magnetostriction value for a given applied field. Under this assumption, the magnetostriction is expressed by the tensor $\boldsymbol{e}\left(e_{x x}, e_{y y}, e_{z z}, e_{x y}, e_{y z}, e_{z x}\right)$. The elastic energy $W_{\text {elastic }}$ and the magnetoelastic energy $W_{\text {magel }}$ are written as

$$
\begin{aligned}
W_{\text {elastic }}= & V \sum_{i}\left[\frac{1}{2} c_{11}\left(e_{x x i}^{\prime 2}+e_{y y i}^{\prime 2}+e_{z z i}^{\prime 2}\right)+\frac{1}{2} c_{44}\left(e_{x y i}^{\prime 2}+e_{y z i}^{\prime 2}+e_{z x i}^{\prime 2}\right)\right. \\
& \left.+c_{12}\left(e_{y y i}^{\prime} e_{z z i}^{\prime}+e_{z z i}^{\prime} e_{x x i}^{\prime}+e_{x x i}^{\prime} e_{y y i}^{\prime}\right)\right], \\
W_{\text {magel }}= & V \sum_{i}\left\{B_{1}\left[e_{x x i}^{\prime}\left(\alpha_{1 i}^{\prime 2}-\frac{1}{3}\right)+e_{y y i}^{\prime}\left(\alpha_{2 i}^{\prime 2}-\frac{1}{3}\right)+e_{z z i}^{\prime}\left(\alpha_{3 i}^{\prime 2}-\frac{1}{3}\right)\right]\right. \\
& \left.+B_{2}\left(e_{x y i}^{\prime} \alpha_{1 i}^{\prime} \alpha_{2 i}^{\prime}+e_{y z i}^{\prime} \alpha_{2 i}^{\prime} \alpha_{3 i}^{\prime}+e_{z x i}^{\prime} \alpha_{3 i}^{\prime} \alpha_{1 i}^{\prime}\right)\right\},
\end{aligned}
$$

where $c_{11}, c_{12}$, and $c_{44}$ are the elastic constants, and $\mathbf{e}_{i}^{\prime}$ is the magnetostriction tensor projected to the crystal axes of the $i$ th grain. Furthermore, $B_{1}$ and $B_{2}$ are the magnetoelastic coupling constants determined from the magnetostrictive constants, $\lambda_{100}$ and $\lambda_{111}$, and the elastic constants. ${ }^{1}$ The total of $W_{\text {elastic }}$ and $W_{\text {magel }}$ is designated as the magnetostrictive energy $W_{\text {striction }}$.

From the above equations, it is clarified that total energy $W_{\text {total }}$ is a function of $\boldsymbol{\alpha}_{i}$ and $\boldsymbol{e}$ for given values of $D$ and applied field. Therefore, we get the simultaneous equations for $\boldsymbol{\alpha}_{i}$ and $\boldsymbol{e}$ by minimization of $W_{\text {total }}$. In our calculation, $\boldsymbol{\alpha}_{i}$ of each grain were determined by using Gilbert's equation. ${ }^{8}$

The assumed material parameters are listed in Table I. Alloys 1-6 are made-up materials to make the discussion more clear.

Figure 2 shows typical $\lambda-H$ loops for $\left(\mathrm{Tb}_{0.3} \mathrm{Dy}_{0.7}\right) \mathrm{Fe}_{2}$, where $\lambda$ and $H$ are the magnetostriction and applied field, respectively. It is clearly seen that soft magnetic properties

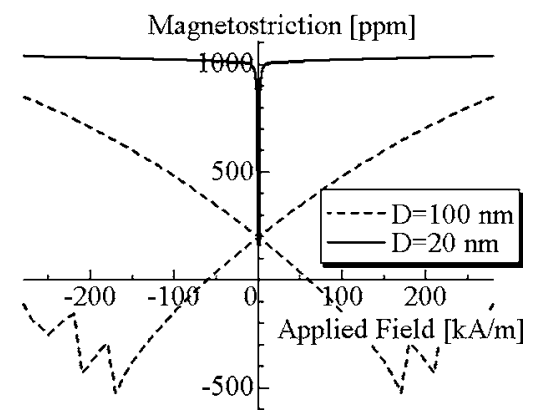

FIG. 2. Magnetostriction vs applied field for a $\left(\mathrm{Tb}_{0.3} \mathrm{Dy} \mathrm{y}_{0.7}\right) \mathrm{Fe}_{2}$ magnet composed by $100 \mathrm{~nm}$ (broken line) and $20 \mathrm{~nm}$ (solid line) grains. depend on the grain size $D$. Therefore, we calculated hysteresis and $\lambda-H$ loops with varying $D$, and the coercivity $H_{c}$ and $(d \lambda / d H)_{\max }$ are shown in Fig. 3 as a function of $D$, together with $H_{c}$ of alloy 1 which is nonmagnetostrictive, where $(d \lambda / d H)_{\max }$ is the maximum value of $d \lambda / d H$ in a $\lambda-H$ loop, and $\lambda_{s}$ is the saturation magnetostrictive constant for polycrystalline material defined as $\lambda_{s}=\left(2 \lambda_{100}+3 \lambda_{111}\right) / 5$. As shown in the figure, $H_{c}$ and $(d \lambda / d H)_{\max }$ vary abruptly at $D$ $=25 \mathrm{~nm}$ for $\left(\mathrm{Tb}_{0.3} \mathrm{Dy}_{0.7}\right) \mathrm{Fe}_{2}$, and soft magnetic properties of $\left(\mathrm{Tb}_{0.3} \mathrm{Dy}_{0.7}\right) \mathrm{Fe}_{2}$ are improved significantly for $D \leqslant 25 \mathrm{~nm}$. On the other hand, $H_{c}$ of alloy 1 varies proportionally to $D^{6}$ between $D=10$ and $25 \mathrm{~nm}$, which agrees with Herzer's predication neglecting magnetostriction. ${ }^{2}$ Consequently, the critical phenomenon indicated for $\left(\mathrm{Tb}_{0.3} \mathrm{Dy}_{0.7}\right) \mathrm{Fe}_{2}$ is expected to originate from magnetostriction.

In order to study the relationship between the magnetostrictive and magnetic anisotropy energies, we calculated $H_{c}$ with varying the magnetostriction and crystalline magnetic anisotropy constants, and the results are shown in Fig. 4 for alloys 2-5 and listed in Table I. For each alloy shown in Fig. $4, \lambda_{s}$ were varied under the condition of the constant $\lambda_{100} / \lambda_{111}$ value which is $90 / 1640$. In addition, we neglected the exchange energy, which corresponds to the assumption of a large enough grain size. As shown in Fig. $4, H_{c}$ increases proportional to $\lambda_{s}^{2}$ and decreases abruptly at a critical $\lambda_{s}$ value. After the abrupt decrease in $H_{c}$, the variation of $H_{c}$ agreed with that of alloy 2 which does not have magnetic anisotropy. The chain line in Fig. 4 is the boundary between two regions: regions I and II. In region II, the effect of the magnetic anisotropy on $H_{c}$ is suppressed by magnetostriction, while $H_{c}$ is affected by a $K_{c 1}$ value in region I.

We compared the magnetic anisotropy energy $W_{a}$ with the magnetostrictive energy $W_{\text {striction }}$, and found that $W_{\text {striction }}$ in the magnetic saturation state is larger than the magnetic

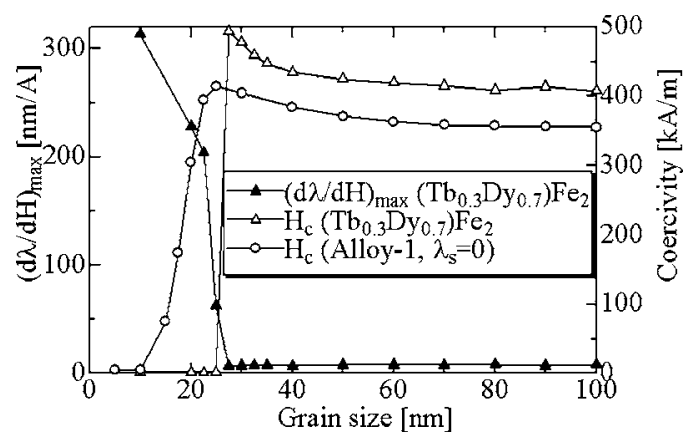

FIG. 3. Grain size dependence of coercivity and $d \lambda / d H$. The open circles indicate the results for alloy 1 . 


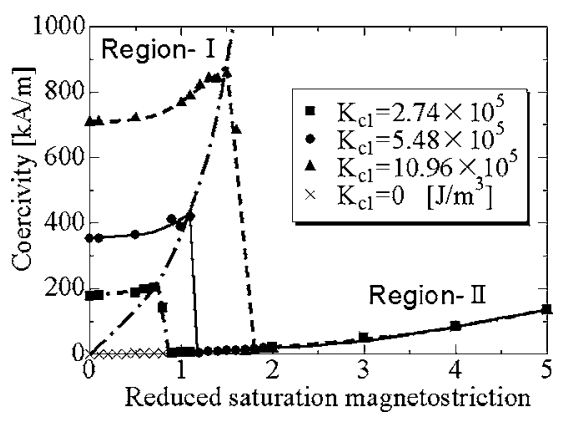

FIG. 4. Dependence of coercivity on saturation magnetostriction $\lambda_{s}$ for alloys $2-5$. $K_{c 1}$ values of these alloys are $0,2.74,5.48$, and 10.96 $\times 10^{5} \mathrm{~J} / \mathrm{m}^{3}$, respectively. The saturation magnetostriction is reduced by the value for $\left(\mathrm{Tb}_{0.3} \mathrm{Dy}_{0.7}\right) \mathrm{Fe}_{2}$ which is $1020 \mathrm{ppm}$.

anisotropy energy in region II. This result resembles the variation of $H_{c}$ of alloy 1 as shown in Fig. 3, for which soft magnetic properties are improved when the exchange energy is dominant to the anisotropy energy.

The variation of soft magnetic properties for alloy 1 , as shown in Fig. 3, can be attributed to the exchange interaction that affects the directions of the neighbor magnetization and aligns them parallel to each other. Considering this effect, the results shown in Figs. 3 and 4 can be explained by assuming a similar effect to the exchange interaction for magnetostriction. Under this assumption, the critical decrease in $H_{c}$ can be explained as follows. In the case of alloy 1 , the exchange interaction has a tendency of aligning the direction of each grain and reduces the "effective" anisotropy energy with decreasing the grain size. In the case of $\left(\mathrm{Tb}_{0.3} \mathrm{Dy}_{0.7}\right) \mathrm{Fe}_{2}$, on the other hand, the magnetostriction boosts the effect of the exchange interaction. Consequently, the magnetostrictive energy becomes dominant to the anisotropy energy at $D$ $=25 \mathrm{~nm}$, and soft magnetic properties are improved significantly.

To confirm the above effect, we calculated $W_{\text {striction }}$ stored in two neighbor grains as shown in Fig. 5. The magnetization of grain 1 was fixed to the [100] direction, and the direction of the magnetization in grain $2, \alpha_{2}$, was varied, and the stored energy was calculated. The result is shown as a function of $\alpha_{2}$ in Fig. 5. In the calculation, we assumed an isotropic magnetostrictive alloy and its material parameters are listed in Table I under alloy 6. As seen in Fig. 5, the minima of $W_{\text {striction }}$ lie down in the [100] and [-100] directions, while the maxima lie perpendicularly to them. This result suggests that the magnetostrictive energy affects the directions of magnetization and aligns them to the same or opposite direction and that it can boost the effect of the exchange energy.

To confirm the above-mentioned effect of magnetostriction for alloys with anisotropic magnetostriction, we calculated the average of $\left|\boldsymbol{\alpha}_{i} \cdot \boldsymbol{\alpha}_{j}\right|$ for all the grains of two alloys which are categorized in alloy 4 and have the same $K_{c 1}$ value and different $\lambda_{s}$ values $\left(\lambda_{s}=510\right.$ and $\left.1530 \mathrm{ppm}\right)$. The calculated average of $\left|\boldsymbol{\alpha}_{i} \cdot \boldsymbol{\alpha}_{j}\right|$ for $\lambda_{s}=1530 \mathrm{ppm}$ was larger than

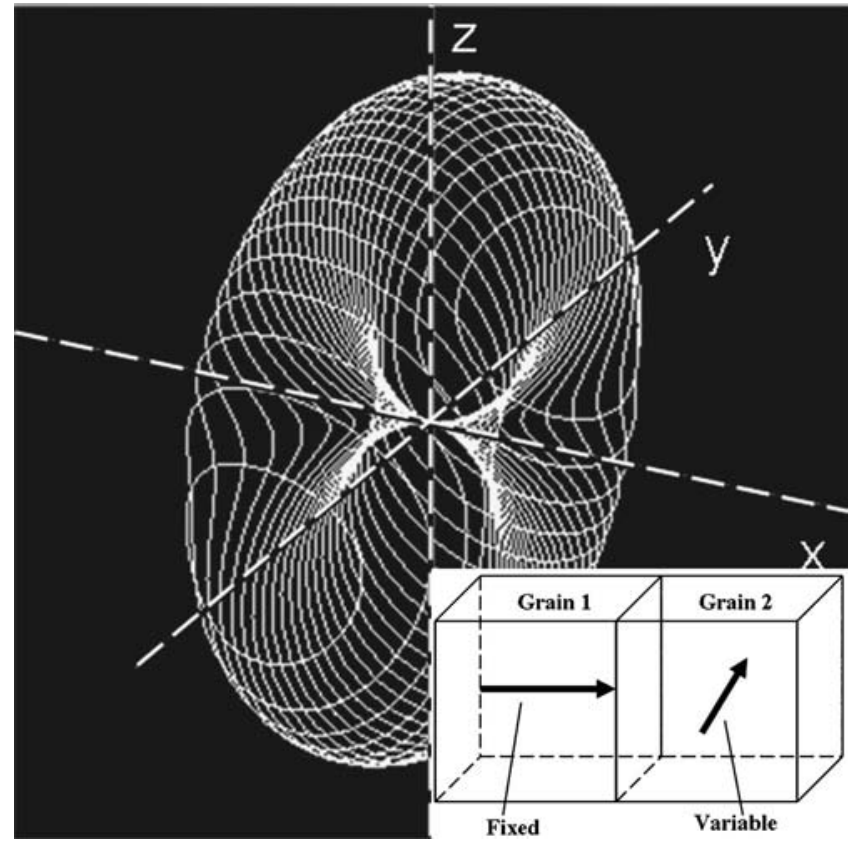

FIG. 5. Calculated distribution of magnetostrictive energy $W_{\text {striction }}$ The energy is shown as a function of the direction of magnetization of grain 2 . The inset shows the calculation model.

that for $\lambda_{s}=510 \mathrm{ppm}$ at any given applied field, which is consistent with the results shown in Fig. 5 and suggests that anisotropic magnetostriction has an effect, that is, aligning magnetization into the same direction.

We have calculated the dependence of magnetostrictive and magnetic properties on grain size and found a unique phenomenon for isotropic materials with giant magnetostriction. Coercivity $H_{c}$ and $d \lambda / d H$ varied abruptly at a critical grain size, and soft magnetic properties were achieved for a grain size smaller than the critical value. This critical phenomenon occurred when the magnetostrictive energy was larger than magnetic anisotropy energy and was explained by the effect that magnetostriction has a tendency of aligning magnetization into the same direction and boosting the effect of exchange interaction.

This work was supported in part by the Ministry of Education, Science, Sports, and Culture of Japan under a Grantin-Aid (Grant No. 16080214).

${ }^{1}$ A. E. Clark, Ferromagnetic Materials, edited by E. P. Wohlfarth (NorthHolland, Amsterdam, 1980), Vol. 1, p. 531.

${ }^{2}$ G. Herzer, IEEE Trans. Magn. 26, 1397 (1990).

${ }^{3}$ Y. Yoshizawa, S. Oguma, and Y. Yamauchi, J. Appl. Phys. 64, 6044 (1988).

${ }^{4}$ B. Winzek, M. Hirscher, and H. Kronmüller, J. Alloys Compd. 283, 78 (1999).

${ }^{5}$ P. Ruuskanen, J. Magn. Magn. Mater. 265, 257 (2003)

${ }^{6}$ N. H. Duc, K. Mackay, J. Bets, and D. Givord, J. Appl. Phys. 87, 834 (2000).

${ }^{7}$ Y. Hayashi, T. Honda, K. I. Arai, K. Ishiyama, and M. Yamaguchi, IEEE Trans. Magn. 29, 3129 (1993).

${ }^{8}$ T. L. Gilbert, Phys. Rev. 100, 1243 (1955). 
Journal of Applied Physics is copyrighted by the American Institute of Physics (AIP). Redistribution of journal material is subject to the AIP online journal license and/or AIP copyright. For more information, see http://ojps.aip.org/japo/japcr/jsp 